\title{
Relation between plaque type, plaque thickness, blood shear stress, and plaque stress in coronary arteries assessed by X-ray Angiography and Intravascular Ultrasound
}

\author{
Simone Balocco ${ }^{a)}$ \\ Department Matemàtica Aplicada i Anàlisi, Universitat de Barcelona, Gran Via 585, 08007 Barcelona, Spain \\ Carlo Gatta \\ Computer Vision Center, 08193 Bellaterra, Spain
}

Marina Alberti

Department Matemàtica Aplicada i Anàlisi, Universitat de Barcelona, Gran Via 585, 08007 Barcelona, Spain

Xavier Carrillo

Hospital Universitari “Germans Trias i Pujol,” 08916 Badalona, Spain

Juan Rigla

Boston Scientific Corporation, Fremont, California 47215

Petia Radeva

Computer Vision Center, 08193 Bellaterra, Spain and Department Matemàtica Aplicada i Anàlisi, Universitat de Barcelona, Gran Via 585, 08007 Barcelona, Spain

(Received 25 May 2012; revised 19 September 2012; accepted for publication 2 October 2012; published 27 November 2012)

\begin{abstract}
Purpose: Atheromatic plaque progression is affected, among others phenomena, by biomechanical, biochemical, and physiological factors. In this paper, the authors introduce a novel framework able to provide both morphological (vessel radius, plaque thickness, and type) and biomechanical (wall shear stress and Von Mises stress) indices of coronary arteries.

Methods: First, the approach reconstructs the three-dimensional morphology of the vessel from intravascular ultrasound (IVUS) and Angiographic sequences, requiring minimal user interaction. Then, a computational pipeline allows to automatically assess fluid-dynamic and mechanical indices. Ten coronary arteries are analyzed illustrating the capabilities of the tool and confirming previous technical and clinical observations.

Results: The relations between the arterial indices obtained by IVUS measurement and simulations have been quantitatively analyzed along the whole surface of the artery, extending the analysis of the coronary arteries shown in previous state of the art studies. Additionally, for the first time in the literature, the framework allows the computation of the membrane stresses using a simplified mechanical model of the arterial wall.

Conclusions: Circumferentially (within a given frame), statistical analysis shows an inverse relation between the wall shear stress and the plaque thickness. At the global level (comparing a frame within the entire vessel), it is observed that heavy plaque accumulations are in general calcified and are located in the areas of the vessel having high wall shear stress. Finally, in their experiments the inverse proportionality between fluid and structural stresses is observed. () 2012 American Association of Physicists in Medicine. [http://dx.doi.org/10.1118/1.4760993]
\end{abstract}

Key words: Intravascular Ultrasound, wall shear stress, wall stress, vascular measurements

\section{INTRODUCTION AND STATE-OF-THE-ART}

Although several epidemiological risk factors have been associated with coronary heart disease (CHD), ${ }^{1}$ several studies suggest that there are additional causes that predispose to arterial disease. In particular, there is an abundant and increasing evidence that the combination of morphological and biomechanical (hemodynamics and vascular stresses) factors play an important role in the initiation and development of atherosclerosis, thus potentially leading to cardiovascular accidents such as plaque rupture. ${ }^{1,2}$
Atheromatic plaque evolves over time and causes plaque grow-up that mainly narrows the arteries and reduces the blood flow. Plaque progression is directly affected by mechanical, biochemical, and biological factors. Researchers made some early attempts to understand associations between these elements with vessel pathology using computational fluid dynamics by modeling the formation and progression of atheromatic plaque. ${ }^{2-4}$ In particular, the analysis of blood velocity and wall shear stress (WSS), associated with geometry, has been recognized to provide early biomarkers of the atherosclerotic plaque formation and growth, ${ }^{5,6}$ while membrane stress and material type are used as indicators of plaque 
fragility. ${ }^{7}$ However, the relation between these indices and the development of different kinds and amount of plaque has not been completely understood. The combined analysis of all these factors, in vivo, can potentially give insights into atherosclerotic plaque evolution and rupture. Such advanced study can be performed intraoperatively on coronary arteries by combining the information obtained from Intravascular Ultrasound (IVUS) and X-ray angiography with computer simulation.

Intravascular ultrasound is a clinical imaging technique providing high-resolution cross-sectional images of coronary arteries. In patients affected by atherosclerosis, IVUS provides valuable information for the study of the atherosclerotic plaque growth. On one hand, it enables the assessment of the severity of the stenosis, ${ }^{8}$ the quantification of the amount of atherosclerotic plaque (plaque thickness), ${ }^{9}$ the assessment of heterogeneous plaque types and the identification of calcified regions, ${ }^{10}$ which are fundamental information for predicting plaque rupture. ${ }^{11}$ On the other hand, the IVUS sequence, when combined with Angiographic projections, provides a three-dimensional (3D) morphological reconstruction of the vessel. ${ }^{12-17}$ Such reconstruction, when coupled to biomechanical computational simulations, leads to the estimation of functional indices such as the blood flow dynamic and the vascular and membrane stress acting on the artery.

The first important contribution in the field has been introduced by Krams et al. ${ }^{18}$ who analyzed the shear stress and the 3D geometry as factors determining the development of atherosclerosis and remodeling in coronary arteries. He employed an IVUS 3D reconstruction technique to calculate the local wall thickness, the principal plane of curvature, and the location of plaque with reference to this plane, relating these results to shear stress in a human right coronary artery in vivo. The analysis of such data leads to the findings that an inverse relationship between wall thickness and shear stress for each velocity level was present, demonstrating that low shear stress promotes atherosclerosis.

In another study, Zhu et al. ${ }^{1}$ showed the relationship between the dynamic geometry and wall thickness of a human coronary artery. Using a multiple regression analysis they showed that: (1) no single dynamic geometry parameter (distance along the vessel, cyclic displacement, axial strain, curvature, and torsion) has a dominant influence on the wall thickness, (2) linear combinations of such parameters predict wall thickness measures with high confidence, and (3) both the time-average values of curvature and torsion and their excursion during the cardiac cycle are positively correlated with maximum wall thickness and cross-sectional asymmetry. However, the use of angiographic images for measuring the vessel pulsation can be unreliable, since this modality might lack of sufficient resolution for a reliable wall tracking in small vessels.

Wahle et al. ${ }^{19}$ proposed a complete and exhaustive analysis of the relation among vascular geometry, hemodynamic wall shear stress, and amount of plaque in the coronary arteries, which is based on a similar 3D reconstruction technique. In his study, the relation between wall shear stress, local curvature, and radial plaque thickness was described. The study first analyzed the dependence of circumferential plaque distribution on the local vessel curvature. Successively, it correlates the plaque distribution and wall shear stress over a set of 48 in vivo vessel segments. The results indicated a stronger correlation of circumferential plaque thickness with local curvature than with wall shear stress. The inverse relationship between local wall shear stress and plaque thickness was significantly more pronounced in vessel cross-sections exhibiting compensatory enlargement (positive remodeling) without luminal narrowing than when the full spectrum of disease severity was considered. The inverse relationship was no longer observed in vessels where the luminal narrowing was inferior to $35 \%$ of vessel cross-sections. The findings in Ref. 19 confirm, in vivo, the hypothesis that relatively lower wall shear stress is associated with early plaque development.

Gijsen et al..$^{20}$ investigated the relationship between shear stress and strain, in human coronary arteries. In their study, the shear stress value was obtained by computational fluid dynamics, while tissue strain was measured by palpography. The conclusions were that low strain is localized in regions with low shear stress downstream of plaques, while higher strain can be found in all other plaque regions, with the highest strain found in regions exposed to the highest shear stresses. The authors concluded that high shear stress might destabilize plaques, which could lead to plaque rupture.

Similar studies have been performed using other imaging modalities. For instance, van der Giessen et al. ${ }^{21}$ presented a framework to explore the relation between wall shear stress and wall thickness, where the vessel centerline is extracted from coronary computed tomography angiography instead of angiographic images. In Ref. 22, the relationship between wall shear stress, plaque thickness, and burden is analyzed in carotid atherosclerotic plaque by means of magnetic resonance images. Moreover three other methods ${ }^{23-25}$ based only on angiographic images can generate 4D coronary arterial trees, i.e., the arterial vessel reconstruction along the cardiac cycles. These techniques are complex and may require further optimization in order to be incorporated into an automatic processing system.

The most recent paper on the topic, proposed by Samady et al. ${ }^{26}$ extended the framework proposed by previous authors introducing the plaque type analysis. In Ref. 26, the authors studied the relation between wall shear stress and the plaque progression (in terms of type and amount of plaque). In the study, 20 patients were monitored both on baseline and follow up exams providing statistics about the evolution of such clinical observations. Samady concluded that segments of the artery having low-WSS are prone to show greater plaque and necrotic core progression and constrictive remodeling. Inversely, segments having high-WSS develop greater necrotic core and calcium progression, regression of fibrous and fibrofatty tissue, and excessive expansive remodeling, which suggests an evolution towards a more vulnerable phenotype. Although this paper was undoubtedly a seminal work in the field, several limitations were present. A first drawback of this study was that the registration between baseline and follow up analysis was manually performed, relating frames extracted from pullbacks of the same patient. As a second limitation, 


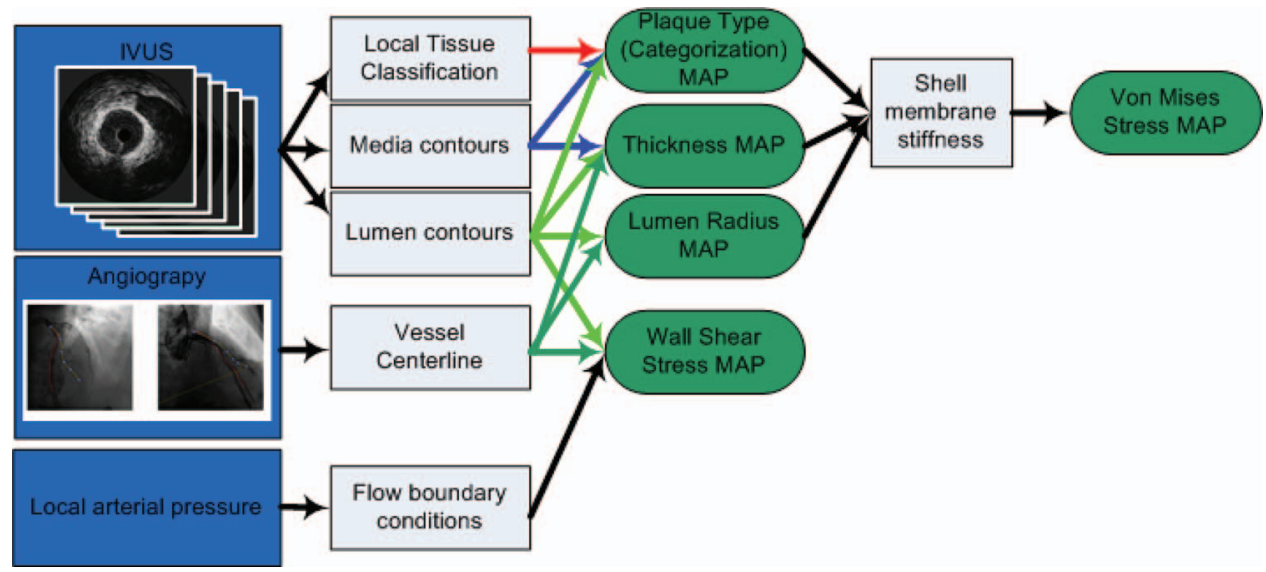

FIG. 1. General scheme of the proposed methodology, depicting the dependence of the morphological, fluid, and structural maps (in red) from the intermediate computations (in gray) and from the physiological measurements (green).

the plaque composition data were derived from the Virtual Histology (VH, Volcano Corporation, Rancho Cordova, CA) analysis performed by the commercial echograph. As claimed by the same authors, the reliability of such tool is questionable, in particular, since little correlation has been found between the necrotic core size identified by the echograph and the histology samples of arteries..$^{26}$ In another paper by Thim et al. ${ }^{27}$ the assessment of necrotic core by virtual histology has been claimed as unreliable. Additionally, the media membrane is not correctly identified by the $\mathrm{VH}$, since all the unclassified areas of the plaque are assigned to this tissue. Besides, being ${ }^{26}$ a clinical paper, few technical details about the reconstruction and simulation technique were provided. The results proposed in Ref. 26 were statistically analyzed; however, the results were averaged over the whole studied population and few details about each single patient analysis have been provided. Regarding the IVUS measurements and the fluid-dynamic study, the computed values were averaged for each cross-section of the artery, thus losing any angular sector information.

In our study, a novel framework for the reconstruction of the artery requiring minimal user interaction is presented. Such reconstruction method is then coupled with a pipeline able to automatically assess morphological (vessel radius, plaque thickness, and type) and biomechanical (fluid-dynamic and structural stresses) maps. Such tool is used to provide qualitative and quantitative analysis of the relation between IVUS measurement and simulations, analyzing both longitudinal and angular sample information, thus extending the analysis of Samady et $a l .{ }^{26}$ In our study, for the first time, the framework allows the computation of structural stresses using a simplified mechanical model of the arterial wall, allowing a preliminary but novel study of the relation between fluid and structural indices. Ten coronary arteries are analyzed illustrating the feasibility of the approach, and confirming clinical observations. A future clinical study, performed over a wider number of patients, will hopefully draw clinically relevant and novel conclusions about the atherosclerotic plaque evolution.
The paper is organized as follows: in Sec. II, the 3D reconstruction method and the computational simulation approach are described. The relations between the morphological, fluiddynamic, and structural indices is discussed in Sec. III. Section IV concludes the paper.

\section{METHOD}

The computational pipeline consists in the threedimensional morphological reconstruction of the vessel coupled to computational simulations.

The volumetric reconstruction of the vessel morphology is obtained fusing IVUS sequences with angiographic projections. A scheme of the approach indicating the data and processing requirements at each step of the pipeline is depicted in Fig. 1. Such approach requires (1) an accurate segmentation of the lumen and media frames belonging to a single phase of the cardiac cycle (end-diastole) in IVUS. Successively, (2) the plaque texture in each IVUS image is characterized using a texture classification method. Finally, the spatial position and orientation of each IVUS frame is obtained analyzing angiographic sequences acquired before and after the IVUS constant speed motorized pullback.

Once the vessel morphology is reconstructed, fluiddynamic and structural indices are computed by finite element simulation.

\section{II.A. IVUS segmentation}

\section{II.A.1. Lumen}

The detection of the arterial luminal area is automatically performed on the IVUS sequence as described in Ref. 28. This technique is particularly suited because all the segmented frames belong to the same cardiac cycle (enddistolic phase). The method first identifies the most stable frames of the pullback by an image-based gating technique. ${ }^{29}$ These frames normally correspond to the images acquired at the end-diastolic phase. Then, the contiguous frames of 
the sequence are registered in order to generate a parametric image discriminating the presence of steady tissues from the blood. Finally, the method segments the vessel lumen by classifying the vessel pixels (lumen vs no-lumen). Indeed, the approach combines temporal information obtained by computing the local correlation between successive frames of the sequence with spatial classification using the Growcut algorithm. ${ }^{30}$

\section{II.A.2. Media-adventitia border}

The media detection in IVUS images is a more challenging task with respect to the lumen segmentation, because of calcium and catheter shadowing artifacts. For this reason, we rely on an improvement of our holistic technique recently proposed in Ref. 31. The method is composed of two phases: first, the different image tissues (media border, lumen, plaques..) are detected by means of a classification technique, then the media border is assessed by considering the relative position of the curve with respect to the surrounding tissues. Hence, the method performs a progressive approximation of the results, combining a first identification of the rough position of the media and a successive local refinement. Both stages consider the relative position of all the tissues in the IVUS image.

As a first step, the classification of the main morphological regions of the vessel in the IVUS image is performed by means of the multiclass stacked sequential learning algorithm. ${ }^{32}$ The classifier is able to discriminate the following areas: blood, fibrotic plaque, lipidic plaque, calcified plaque, media, adventitia, guide-wire, shading, and external tissue. The tissue classification algorithm is based on a similar technique as the one described in Ref. 10 where the approach is validated against histological data.

In the second part of the method, the points labeled as media are used as initial information with the aim of optimally fit a continuous and smooth curve respecting the spatial relative position between the tissues previously obtained (see Ref. 31 for more details). As a result of this approach, both media border and arterial tissue properties comprised between lumen and media contours, are provided. The method is applied only to the gated frames previously identified by the gating technique, so that the processed images are all in the same cardiac phase.

\section{II.A.3. Plaque thickness and categorization}

The lumen and media contours obtained applying the described technique are used to delimit a region of the image in which heterogeneous plaques types and media membrane might be present. Figure 2(a) illustrates a frames of a pullback in polar view. The lumen and media contours are superimposed to the grayscale IVUS image and to the corresponding tissue classification (columns). For each frame, the plaque thickness is computed as the radial distance (computed along each polar line) between the lumen and the media.
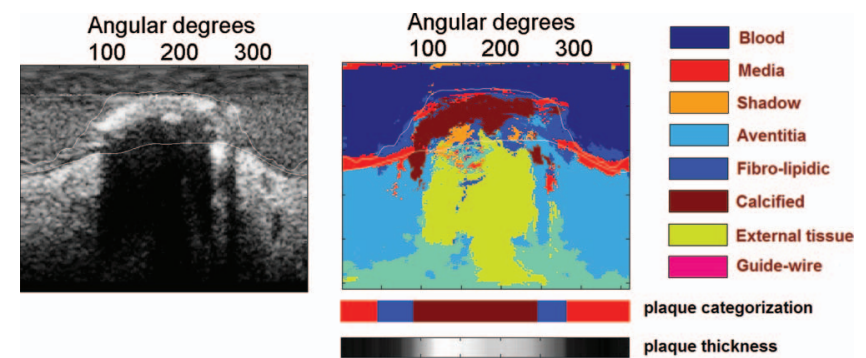

(a)
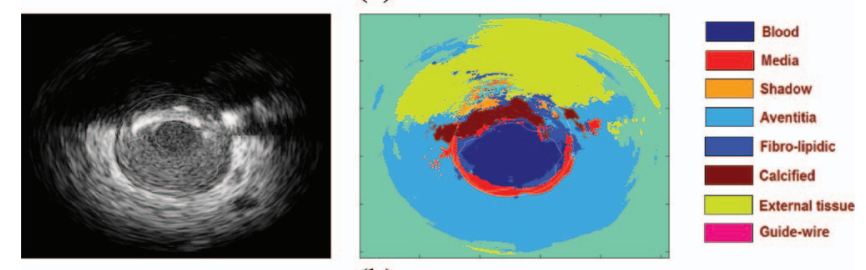

(b)

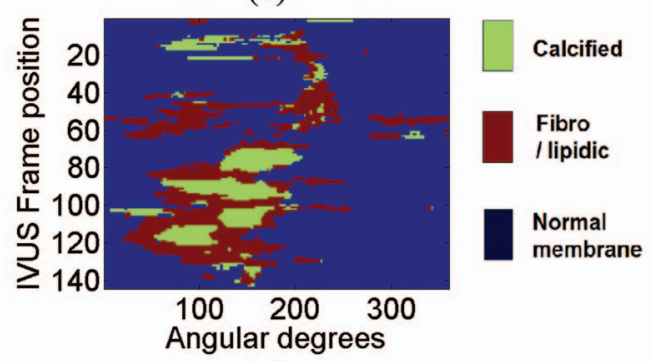

(c)

FIG. 2. (a) Example of one segmented (left) and classified (right) frame. The classification is translated in an angular categorization of three classes (horizontal bar below the classified frame), discriminating media, fibro-lipidic, and calcified angular sectors (first bottom bar). The second bottom bar represents the plaque thickness in gray values (the brighter, the higher). (b) The same IVUS image and classification map in Cartesian coordinates. (c) Tissue categorization for the whole pullback.

In this preliminary analysis of the relation of the tissue types with the other vascular properties, we are interested in obtaining the most robust estimation of the circumferential material properties, so as to derive statistically reliable and meaningful conclusions. For this reason, the information provided by the plaque characterization algorithm ${ }^{10}$ was not fully exploited but a successive step called tissue categorization [Fig. 2(a) (bottom)], which summarizes the predominant tissue contained in the area between the two outlined contours. This is done by computing the mode of the tissue types obtained by the tissue classification algorithm for each radial sector. The computation of the mode reduces possible plaque characterization errors and homogenizes the tissue regions, making the plaque analysis stage more reliable.

In the present study, in order to obtain consistent statistics, and evaluate the results on a sufficiently high number of samples, the lipidic and fibrotic samples have been combined in a single region, since in the data-set, the lipidic samples were few, inhomogeneous and scattered into the fibrotic area (no lipidic pools were present). However, it is important to note that such two tissues can be discriminated by the system in other data-sets containing lipid accumulations, given that the tissue characterization algorithm correctly classifies both classes. Instead of incorrectly classifying the pixels 


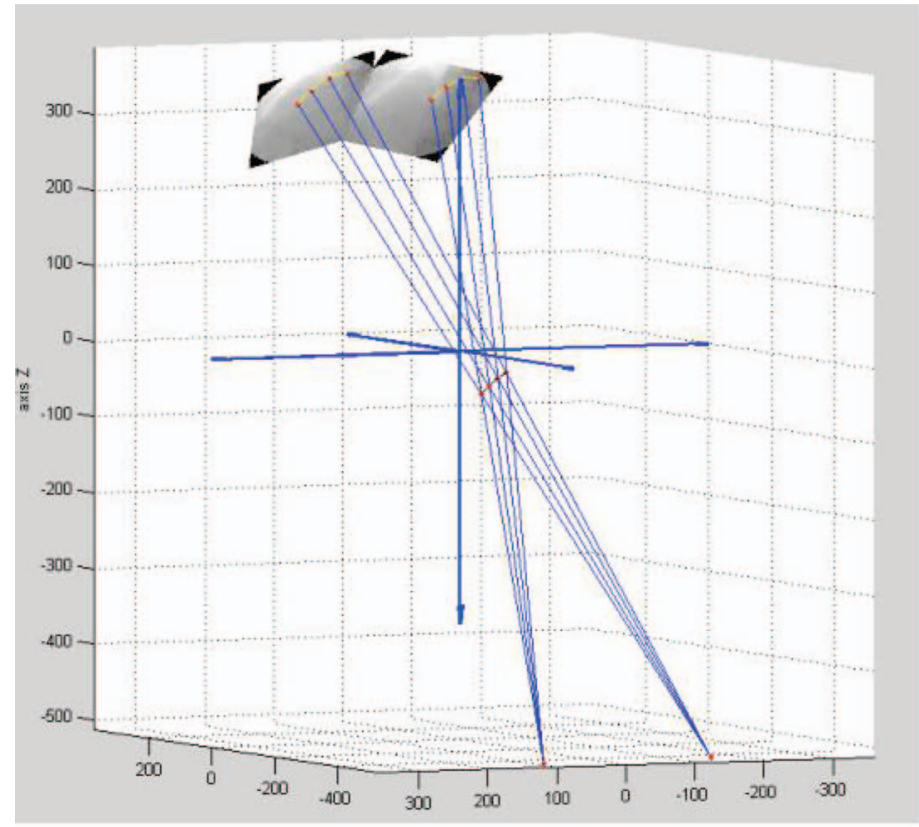

(a)
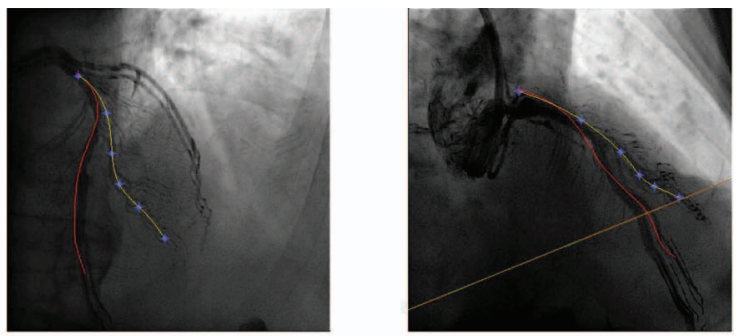

(b)

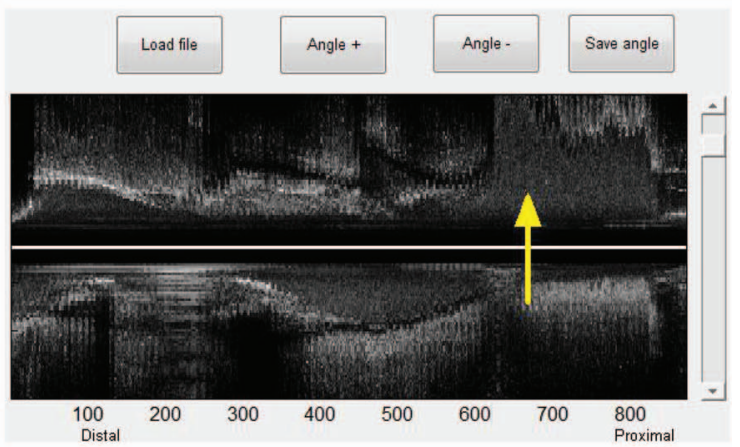

(c)

FIG. 3. (a) Angiographic projections located in a global reference system with its origin in the isocenter of both image projections. (b) Interpolating 3D spline fitted to the catheter path (in red) and to the bifurcation branch (in yellow) of each projection. The blue crosses indicate the control points which can be displaced along the epipolar line (green). (c) User interface allowing the selection of the rotation angle allowing to visualize the bifurcation branch selected in (b). The yellow arrow shows the position of the selected bifurcation, and the artery is displayed from distal (left) to proximal (right).

belonging to the catheter artifact and the shadow behind the calcification, ${ }^{27}$ such regions were identified by the classifier and discarded from the categorization process, exploiting the holistic contextual approach proposed by Ciompi et al. ${ }^{31}$

As a result, the proposed algorithm, discriminates if the areas of the vessel contains healthy membrane or plaque, and if the plaque is mainly calcified or fibrotic. Although only circumferential samples of material properties are assessed using this approach (leading to a simplification of the heterogeneity of the plaque contents), the analysis proposed in this paper is locally more detailed than in Ref. 26 where statistics only of the whole frame (and not local circumferential sample assessment) have been reported. The richer information provided by the tissue classification (plaque radial distance, presence of lipidic pools, and heterogeneity of the tissue) will be exploited in a future study.

Figures 2(a) and 2(b) illustrates the plaque thickness and characterization estimated on an exemplar frame. On the bottom of Fig. 2(a), a tissue categorization result is illustrated. Finally, Fig. 2(c) shows the resulting categorization of an IVUS pullback, summarizing, for each frame, and each angle, which areas of the vessel are affected by plaque and if such plaque is calcified or fibrotic.

\section{II.B. Angiography: IVUS fusion}

\section{II.B.1. Image fusion}

The morphological reconstruction of the vessel is assessed by reconstructing the tri-dimensional vessel path, obtained by coupling the IVUS sequence with the corresponding angio- graphic projections. ${ }^{17}$ Three pairs of angiographic sequences (each of them lasting at least one cardiac cycle) are required. For each couple of acquisitions, the two projection angles are chosen in order to ensure at least $40^{\circ}$ between them [Fig. 3(a)]. The first two pairs of acquisitions (Angio-land Angio-2) allow the reconstruction of the IVUS catheter path, while a third pair (Angio-3) is used to visualize the coronary branching and requires contrast injection. The first pair of sequences is acquired when the catheter is completely deployed, the second and the third when the pullback procedure is completed [Fig. 3(b)]. The acquisitions are performed using a monoplanar $\mathrm{x}$-ray imaging system, ensuring that no table translation occurred among the recordings. All the x-ray sequences are ECG gated (end-diastole) in order to assure the synchronization between the acquisitions. The patient breathing may cause only rigid translation which does not affect the quality of the reconstruction.

The reconstruction procedure consists in two phases: first, using Angio-1 and Angio-2, a global reference system with its origin in the isocenter of the image projections is established. The catheter tip is identified by the user, and from that point the catheter segmentation is performed using the fast marching algorithm ${ }^{33}$ computed in both angiographic projections of the IVUS catheter. The catheter pathis reconstructed following the methodology proposed by Dumay et al. ${ }^{17} \mathrm{~A}$ biplanar spline controlled by $n$ control points is fitted to the data following the undistortion corrections proposed in Ref. 34 [Fig. 3(b)]. In our experiments, setting $n$ equal to five provided acceptable fitting results over the whole data-set. However, such value can be modified by the user or automatically set based on the vessel length and its complexity. If needed, 
the final 3D path can be manually corrected by displacing the control points along the epipolar line [Fig. 3(b) green line]. This global system allows establishing exact correspondence between the segmented catheter paths of both projections and, consequently, recover the real 3D path that the catheter followed during its pullback inside the blood vessel.

As a second phase, the angular rotation of the 3D local reference system (Frenet-Serret frame) with respect to the catheter path is estimated by computing the angle between the main artery and a second trajectory (bifurcation path) corresponding to a bifurcation artery. The bifurcation path is reconstructed by manually indicating an extreme of one branching artery and applying again the fast marching algorithm ${ }^{33}$ until intersecting the catheter path [Fig. 3(b)]. Successively, the user identifies the corresponding torsion by selecting the angle in which the bifurcation is visible on the IVUS long view image [Fig. 3(c)]. The manual identification of the branching leads to a robust identification of the vessel orientation, since it exploits reliable physiological landmarks, reducing the risk of computational errors. However, this last operation could be automatically performed, for instance, exploiting algorithms for automatic bifurcation identification in IVUS (Ref. 35) and angiographic images ${ }^{36}$ or using the automatic optimization procedure described in Ref. 19.

The reconstruction performed in the paper uses a single bifurcation (chosen in general as the largest or the most visible, having the minimum foreshortening and overlap by the main vessel). However, multiple bifurcation paths can be drawn by the user in order to increase the number of manual landmarks used for the angle estimation.

The adjustment of rotational angles based on side branches ensures that only a relatively short segment near the bifurcation is aligned. In order to compensate for the potential catheter rotations that could generate axis torsion, the successive IVUS frames are aligned using a preprocessing algorithm ${ }^{37}$ which has been demonstrated to be effective for reducing the catheter rotation due to vessel tortuosity.

\section{II.B.2. Mesh reconstruction}

The triangular mesh describing the inner surface of the vessel in end-diastole has been generated using the lumen segmentation contours described above. For each gated frame of the pullback, the segmented contour has been sampled in $N c$ contour points (Fig. 4), and connected using B-splines to the neighboring frames ensuring the minimal torsion between the contours points (thus the successive orientations of the FrenetSerret frame). The variable $N c$ is an arbitrary value which defines the circumferential resolution of the vessel contour and can be set by the user; in this paper, it is empirically set to 22 as a trade off between computational efficiency and local shape accuracy.

The morphological values computed so far (such as the vessel and plaque thickness, and the tissue properties assessed as described in Sec. II.A.3) are assigned to each triangle of the vessel surface mesh (Fig. 1), called Thickness MAP and Plaque Type MAP. Since each IVUS frame is locally oriented according to the Frenet frame on the vessel centreline, such

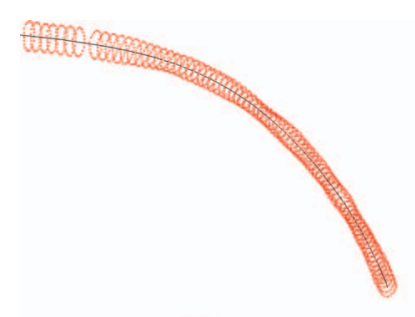

(a)

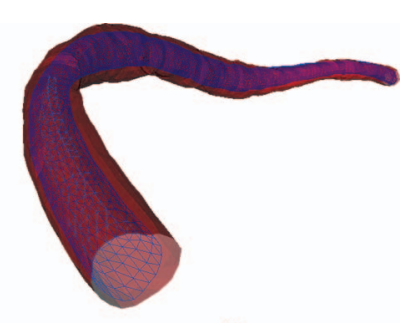

(b)
FIG. 4. (a) Segmented contour in each gated frame of the pullback, along with the 3D catheter path. It can be noted that the distance between the contours is irregular since it depends on the patient heartbeat. (b) 3D mesh reconstruction of lumen (blue mesh) and media (red mesh).

meshes summarize, per each radial sector, the thickness and the predominant plaque type of each slice in the perpendicular direction with respect to the vessel axis.

In order to compute fluid and structural arterial values, the finite-element software (COMSOL Multiphysics $\AA$ v3.5, Burlington, MA) provides a volumetric mesh generator, which automatically refines and adjusts the triangular mesh, and generates volumetric quad-element mesh optimized for finite-element computation.

\section{II.C. Biomechanical model}

The arterial stresses are strongly influenced by the vessel geometry, by the vessel material properties, and by the physiological boundary conditions. This section introduces the morphological and biomechanical models used to compute the fluid and structural stresses together with the model parameters.

\section{II.C.1. Fluid analysis}

In order to compute the flow dynamic in each location of the vessel, the blood is modeled as an incompressible Newtonian fluid ${ }^{19,38}$ using the Navier-Stokes equation

$$
\rho \frac{\partial v}{\partial t}-\nabla \eta\left(\nabla v+(\nabla v)^{T}\right)+\rho(\nabla v) v+\nabla p=0,
$$

where $v$ is the time-dependent flow velocity in the vessel, $p$ is the hydraulic pressure, $\rho$ is the mass density, $\eta$ is the kinematic viscosity of the blood, and ${ }^{T}$ is the transposition operation.

The value of the WSS at each point of the vessel boundary can be computed as

$$
\tau_{w}=\rho \eta\left(\frac{\partial u}{\partial r}\right)_{r=0},
$$

where $u$ is the flow velocity parallel to the wall and $r$ is the distance to the wall.

Since the lumen segmentation has been performed at the end-diastolic phase, corresponding boundary conditions are assumed..$^{38}$ The steady state simulations have been performed considering the diastolic inflow pressure locally measured by pressure catheter inserted into the ostium area of the coronary artery, while an outflow open boundary condition pressure is set, thus letting the simulator to compute the corresponding 
velocity flow and relative pressure drop inside of the vessel domain.

It must be noticed that the vessel branches are not reconstructed by the algorithm, hence introducing cumulative fluid-dynamic error distally. Such problem could be reduced employing a vessel-branching meshing technique similar to Ref. 39

\section{II.C.2. Structural analysis}

Under the assumption that the coronary artery undergoes to small and slow deformations at around the end-diastolic phase, the membrane behavior has been defined by the Hooke law, whose most general form for isotropic materials relates the stress $\left(\sigma_{i j}\right)$ and strain $\left(\varepsilon_{i j}\right)$ tensors as

$$
\sigma_{i j}=3 K\left(\frac{1}{3} \varepsilon_{k k} \delta_{i j}\right)+2 G\left(\varepsilon_{i j}-\frac{1}{3} \varepsilon_{k k} \delta_{i j}\right)
$$

being $K$ and $G$, respectively, the bulk modulus and the shear modulus, which are constant depending on the material and $\delta_{i j}$ is the Kronecker delta.

In order to visualize the stress tensor, the Von Mises stress, ${ }^{3,4}$ which is a scalar intensity summarizing the amount of stress in the material, is calculated as follows:

$$
\sigma_{V M}=\sqrt{\sigma_{11}^{2}+\sigma_{22}^{2}+\sigma_{33}^{2}-\sigma_{11} \sigma_{22}-\sigma_{22} \sigma_{33}-\sigma_{33} \sigma_{11}+3 \sigma_{12}^{2}+3 \sigma_{23}^{2}+3 \sigma_{31}^{2}} .
$$

Regarding the material modeling, instead of postulating the membrane as a volumetric domain enclosed between the lumen and media meshes, in this paper, we chose to approximate the vessel as a shell object. ${ }^{40}$ In the proposed model, the vessel membrane is defined by a triangular surface composed by Argyris elements, ${ }^{41}$ in which the thickness and the material properties are variable for each mesh element. Such solution provides two advantages with respect to the classical volumetric modeling: (1) the computational time is extremely reduced, while maintaining an acceptable approximation of the results in a static domain ${ }^{40}$ and (2) it does not require a radial tissue classification of the material properties but simply the estimation of the dominant tissue in each surface mesh element. As a consequence, the stiffness of a shell element is a surrogate of elasticity, which represents the composite stiffness of the arterial membrane and plaque in the normal direction of the vessel, and it is obtained as the product between the Thickness MAP and the Young modulus of each tissue assigned to the Tissue Type MAP.

The Young modulus values assigned to each plaque were based on the general average of compiled data taken from multiple studies on atherosclerotic tissues. ${ }^{4,42}$ Similar to Ref. 4, the calcium value was assumed equal to $2000 \mathrm{kPa}$. A value of $1500 \mathrm{kPa}$ was assigned to the fibro-lipidic plaque as reported in Ref. 42, thus considering this medium as mostly composed by fibrotic tissue, while the elasticity of the arterial membrane was set to $1000 \mathrm{kPa}^{42}$ All the tissues were considered linear elastic, isotropic plane-strain, and nearly incompressible.

\section{II.C.3. Finite element simulation}

The fluid-dynamic and structural equations of the biomechanical model are solved by finite-element analysis. The computation provides two additional maps: the Wall Shear Stress MAP and the Von Mises Stress MAP which represent, respectively, the friction stresses of the blood and the structural stress into the arterial membrane. Besides, the software provides additional fluid-dynamic indices, such as the flow velocity and streamlines (Fig. 5), which can be exploited in future studies for evaluating the fluid-dynamic patterns in the stenotic areas of the artery. ${ }^{38}$

\section{RESULTS}

Using the described framework, morphological, fluiddynamic, and structural maps belonging to ten arteries have been qualitatively and quantitatively analyzed. Regarding the quantitative study, since several relations among those indices have been extensively described in previous studies, we present only a series of experiments extending previous analysis or introducing novel findings. The results are presented separately for each artery, in order to corroborate if the relation between morphological and biomechanical factors is confirmed on all the arteries, since the average statistics over the whole data set might mask possible outliers.

\section{III.A. Materials}

Ten IVUS pullback (about 2000 frames long each) belonging to different patients acquired prior to stent implantation have been analyzed. The patient population is composed by two females and eight males ranging from 41 to 77 years, with an average age of 63 years, all of them presenting cardiovascular risk factors. Four patients were affected by stable angina, five by non-ST elevation myocardial infarction, and one with ST acute coronary syndrome. All the enrolled patients required invasive IVUS evaluation and were found to have mild nonobstructive lesions. The patients plaques were randomly chosen, i.e., vessels under evaluation included early-stage atheromas with lumen geometry retained by 

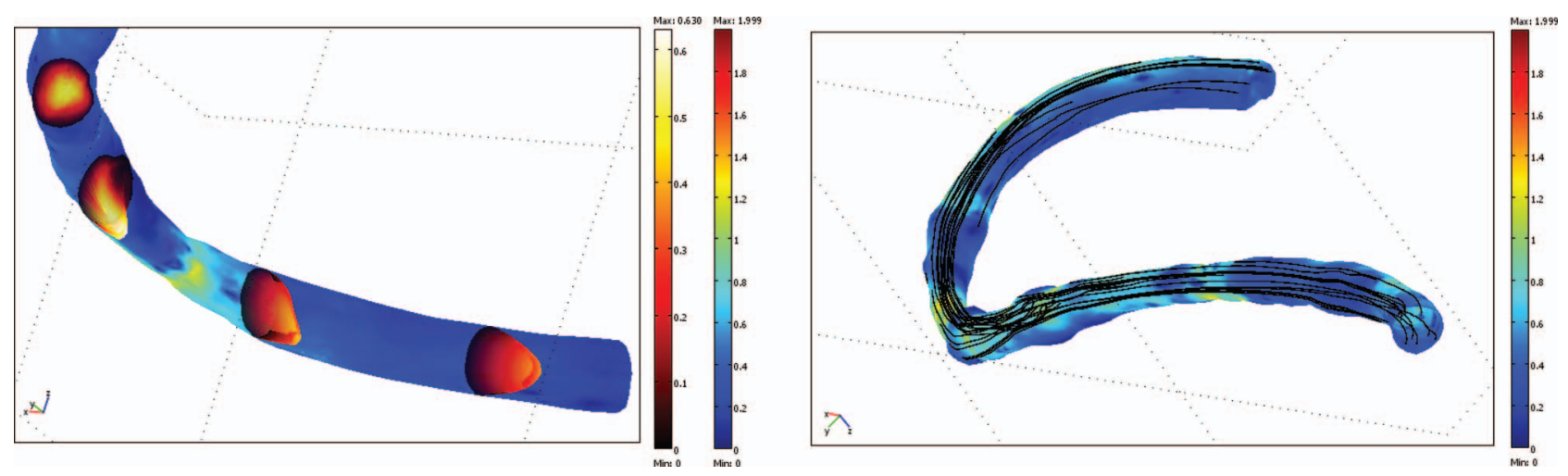

FIG. 5. Evaluation of fluid-dynamic patterns in one of the analyzed arteries (case \#2). On the left picture the flow profile (in red colormap) is superimposed to the wall shear stress map (rainbow colormap) illustrating the velocity changes from parabolic to asymmetric profile. On the right picture, the flow streamlines are superimposed to the wall shear stress distribution in order to visualize the areas where the blood impacts on the wall and possible recirculation areas downstream to a plaque.

compensatory (positive) remodeling up to advanced lesions with luminal narrowing.

In these experiments, no patients presented highly irregular heart beat or tachycardia. Eligible patients provided written informed consent. The acquisitions have been performed using an IVUS Galaxy II System with a catheter Atlantis SR Pro $40 \mathrm{MHz}$ (Boston Scientific). The ten arterial segments have been reconstructed as described in Sec. II.B.2 and it results in superficial meshes with a number of triangles ranging from 2268 to 2624 elements, and a volumetric mesh with a number of elements ranging from 15931 to 22389 elements.

\section{III.B. Qualitative evaluation}

In this section, the relation between several maps computed along the surface of the vessel representing the lumen radius, plaque thickness, plaque type, fluid stress, and vessel stress respectively, can be qualitatively appreciated. Figure 6 summarizes the results obtained from the computations.

With the aim of showing demonstrative images on the relation between the analyzed parameters, three pictures of exemplar arteries have been magnified in Fig. 7, in order to facilitate the visualization of the most interesting areas, i.e., in correspondence of large calcifications, stenoses, and accumulation of stresses. In Figs. 6 and 7, several relations can be observed circumferentially (within a given section of the artery) and globally (comparing a section within the entire vessel). As already demonstrated numerically in Ref. 19, observing a circumferential section of the artery, the higher plaque thickness is in general located on the wall having concave curvature (Fig. 7, first two rows) and in correspondence to extended calcification. Again circumferentially, comparing the second and third rows of Fig. 7, it can be observed that the fluid shear stresses are located in the opposite wall of the plaque deposits, confirming that the plaque deposit is higher in regions characterized by a low shear stress. When the artery is globally analyzed, it can be noticed in the first and third rows of Fig. 7, that mild wall shear stress can be found in fibrolipidic areas, while the maximum blood stresses are adjacent to stenoses generated by calcified plaques. Additionally, observing globally the first and the second rows of Fig. 7, heavy calcifications correspond to regions having high plaque burden. Globally, the membrane stress distribution (Von Mises stress), which depends on the plaque thickness and the plaque characteristics by definition, results inversely proportional to the blood shear stress. These findings are in agreement with the results shown in the previous studies, ${ }^{1,18,19,22,26}$ and this qualitative evaluation has been corroborated in Sec. III.C by quantitative statistical evaluations.

\section{III.C. Quantitative measurements}

In this section, the statistical relation between morphological, fluid, and structural values, computed over the arterial maps, is quantified. In each section of the vessel, $N c$ samples have been recorded for each variable.

Since the data distribution does not follow a specific model (for instance, Gaussian), a statistical significance test of each category would not provide any additional information with respect to the box-plot analysis (which reports the median, first quartile, third quartile, minimum, maximum, and outliers). However, in each experiments, tables illustrating the mean and standard deviation of each experiments are also included.

\section{III.C.1. Wall shear stress vs plaque thickness}

As a first experiment, the relation between plaque thickness and fluid stresses has been locally analyzed in each transversal cross-section of the vessel. Similar to Ref. 26, we reported the plaque thickness values separated according to very low $(0 \%-25 \%)$, intermediate $(25 \%-50 \%)$, high $(50 \%-$ $75 \%$ ), and peak (75\%-100\%) WSS values. Obviously, the absolute value of the wall shear stress depends on the blood 

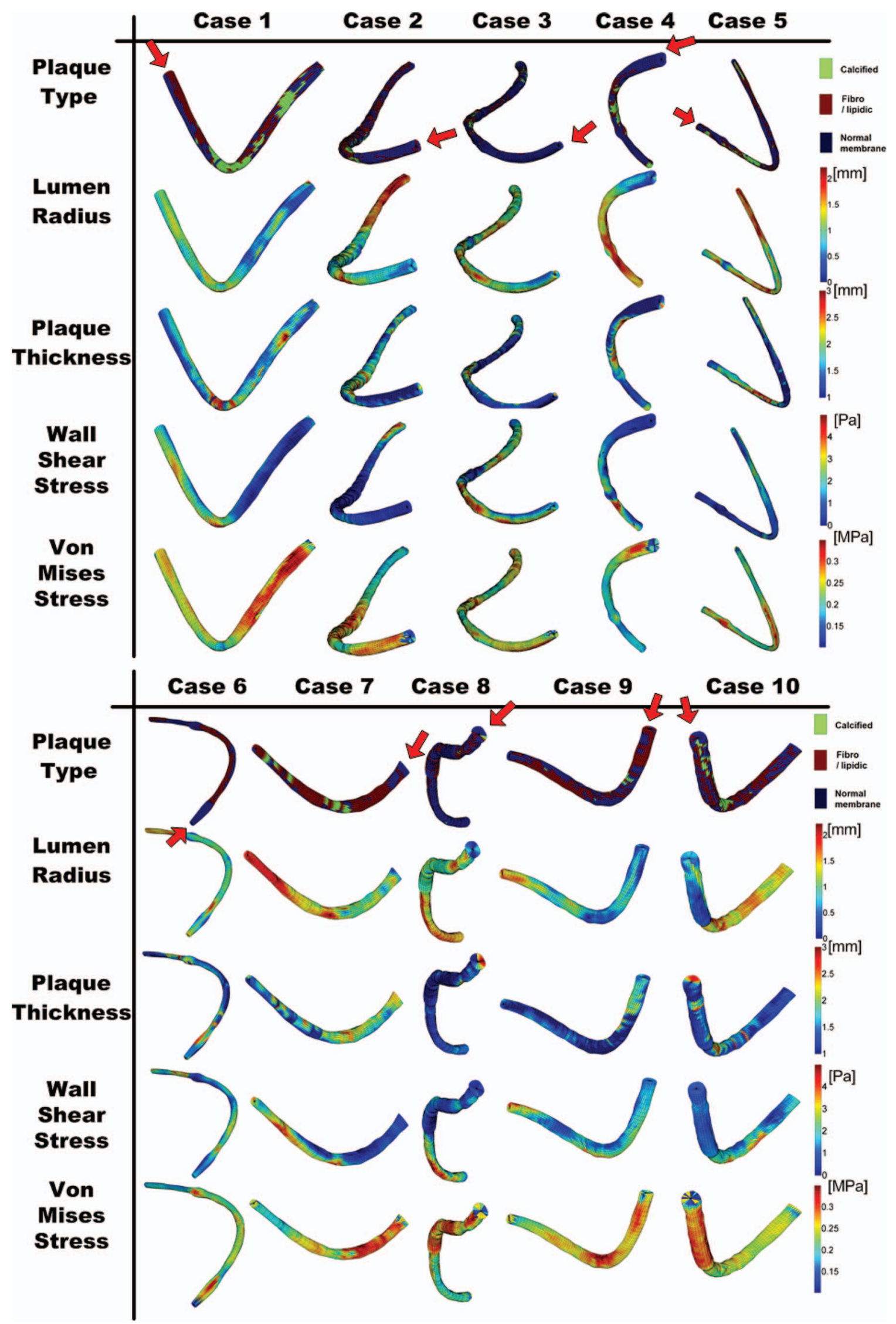

FIG. 6. Surface triangular meshes for the ten analyzed coronary arteries. The color intensities represent, respectively, the plaque type (first and third rows), lumen radius (second and seventh row), the plaque thickness (third and eighth rows), the wall shear stress (fourth and ninth rows) and Von Mises stress (fifth and tenth rows). The color-bar representing the color intensity used in all the figures of the same row is shown on the right. The arteries are oriented in order to optimally show both high WSS and plaque thickness areas. A red arrow indicates the flow direction (hence the proximal and distal parts of the artery). 

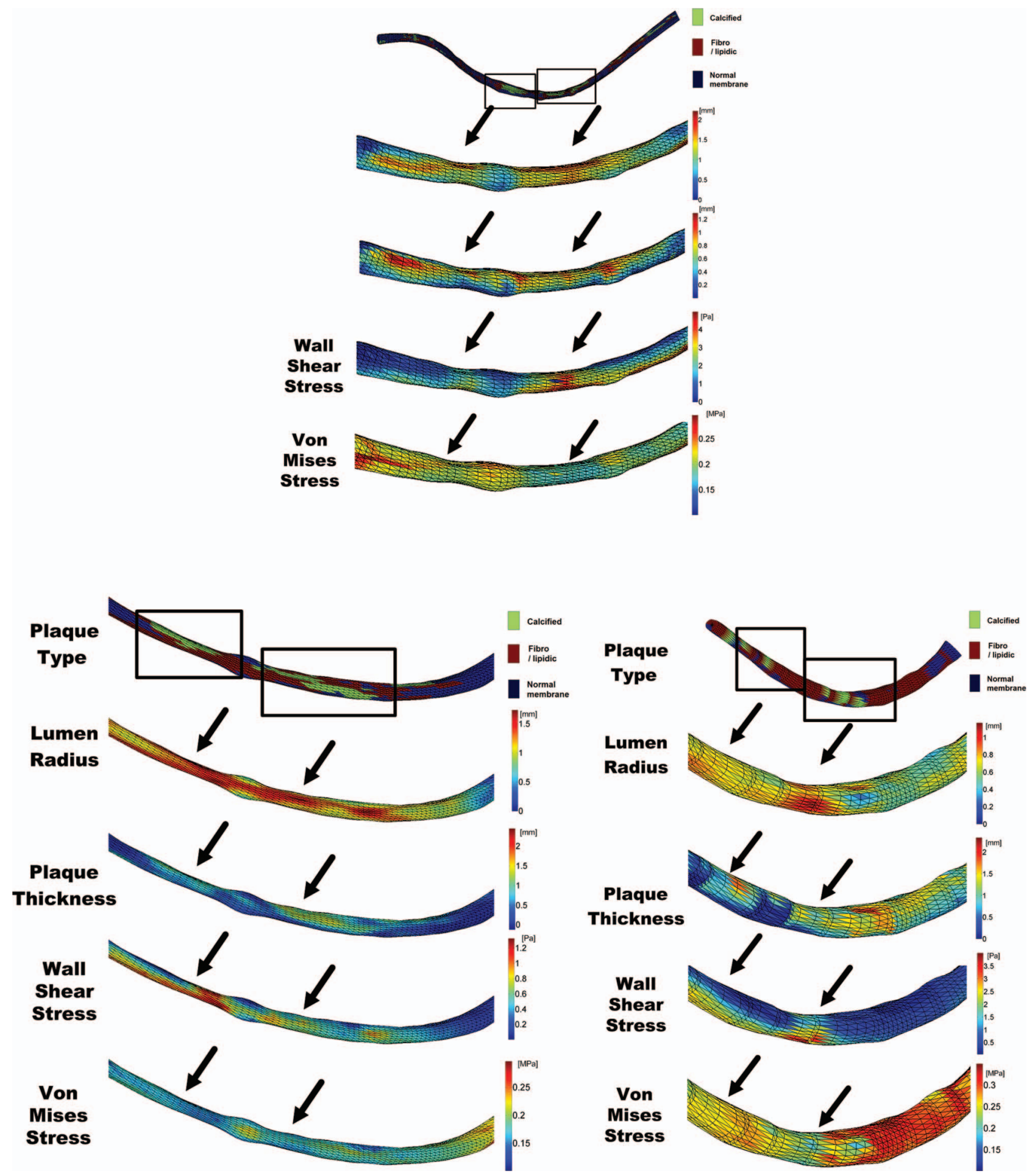

FIG. 7. Details of Fig. 6, illustrating the computed morphological, fluid, and structural maps: plaque type (first row), lumen radius (second row), plaque thickness (third row), wall shear stress (forth row), and Von Mises stress (fifth row). The black arrows point out interesting location showing the relation between the calcified regions (black boxes) with the high stress areas, in general, located close to a stenosis.

velocity and the vessel local radius, and thus it is specific for each patient physiology, and on the vessel location (how distal the IVUS acquisition has been performed) as it can be observed in Fig. 6. Hence, the values in Fig. 8 and in Table I have been normalized in the range [ $\left[\begin{array}{ll}0 & 1\end{array}\right]$ independently for each patient, to stress the plaque thickness/WSS relation, while the maps for each artery are shown with absolute values in Fig. 6. As demonstrated by the box-plots in Fig. 8, the plaque thickness is inversely proportional to the circumferential wall shear stress for each cross section of the vessel, confirming the findings in Refs. 19 and 22. Such findings confirm the interest of considering the spatial samples of each 

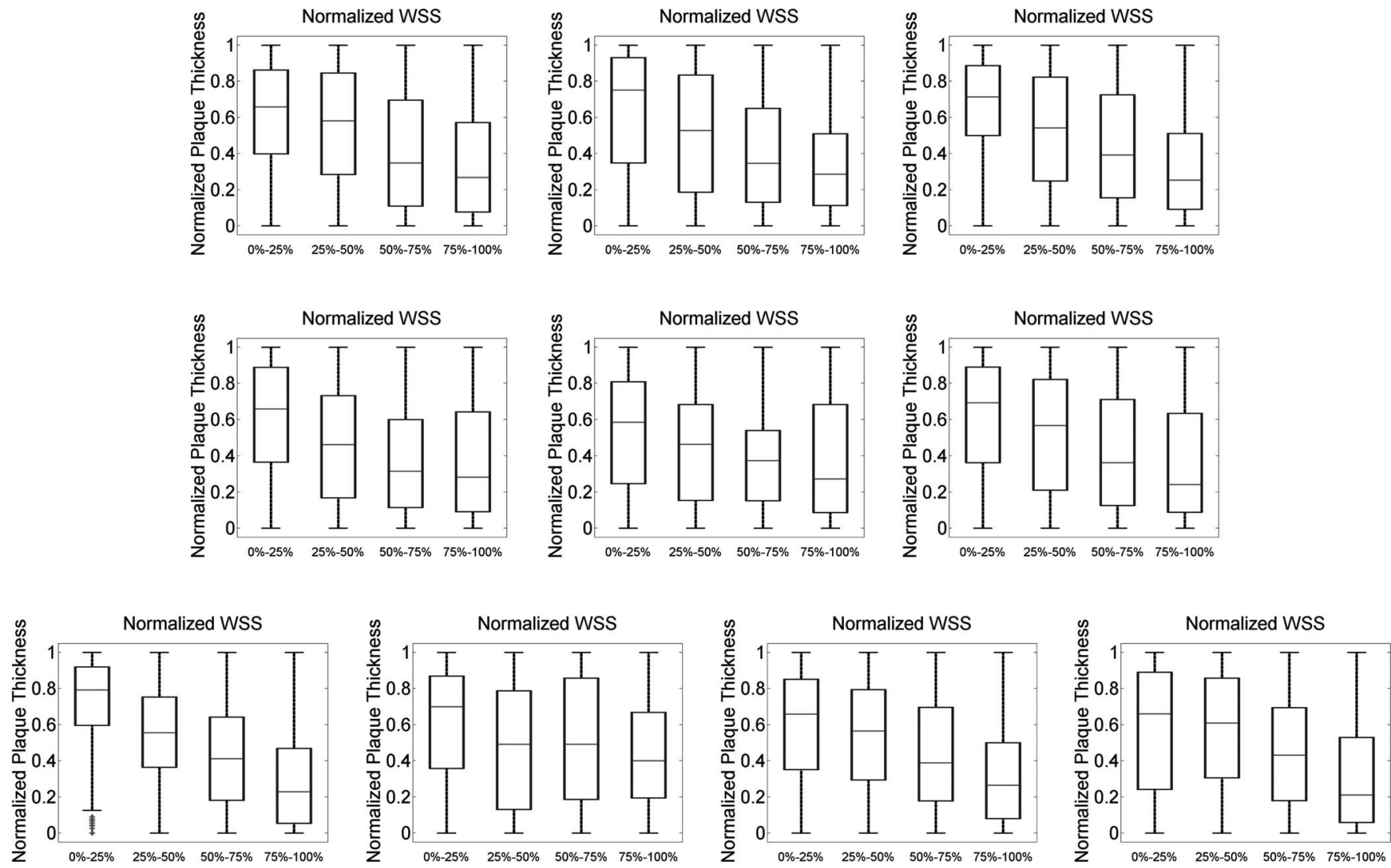

FIG. 8. Normalized plaque thickness vs normalized wall shear stress analyzed in each transversal cross-section of the artery.

cross-section independently, instead of averaging the results by cross-sections as done in Ref. 26.

\section{III.C.2. Plaque thickness vs plaque type}

The relation between the amount of plaque and tissue type has been globally analyzed by Samady et $a l^{26}$ They reported that for each slice, fibro-lipidic tissue is the most prominent is the area, followed by the necrotic, by the lipidic and finally by the calcified areas, and this observation is confirmed for low, medium, and high WSS values. Such analysis can be improved in several ways. First of all in Ref. 26, the plaque information is not fully exploited, since the classification technique available in the commercial echograph $(\mathrm{VH})$ assigns all the unclassified pixels of the plaque (which can occupy up to $40 \%$ of the plaque area) to the media tissue. ${ }^{43}$ As a con- sequence, on one hand not all the pixels of the plaque are considered; on the other hand, the WSS values are not analyzed in the regions belonging to the healthy membrane. ${ }^{43}$ Regarding the necrotic plaque classification, the classification of such area is questionable. ${ }^{26}$

As a second drawback, the measured areas were averaged along each cross-section of the artery and on the whole dataset, thus losing any local information. When we performed a similar analysis on our data-set (but measuring the plaque thickness instead of plaque area) and averaging the results per cross-sections, a similar hierarchy of the plaque distribution was found, with a plaque thickness of $1.25 \mathrm{~mm} \pm 0.21 \mathrm{~mm}$, $0.83 \mathrm{~mm} \pm 0.15 \mathrm{~mm}$, and $0.55 \mathrm{~mm} \pm 0.11 \mathrm{~mm}$, respectively, for the fibrotic, calcified, and normal membrane regions, thus confirming the same findings of Samady et al. ${ }^{26}$

However, it is interesting to note that when each circular sample is separately analyzed, instead of averaging

TABLE I. Normalized plaque thickness vs normalized wall shear stress analyzed in each transversal cross-section of the artery (mean and std).

\begin{tabular}{|c|c|c|c|c|c|c|c|c|c|c|c|c|c|c|c|c|c|c|c|c|}
\hline \multirow{2}{*}{$\frac{\text { Case }}{0 \%-25 \%}$} & \multicolumn{2}{|c|}{$\# 1$} & \multicolumn{2}{|c|}{$\# 2$} & \multicolumn{2}{|c|}{ \#3 } & \multicolumn{2}{|c|}{$\# 4$} & \multicolumn{2}{|c|}{ \#5 } & \multicolumn{2}{|c|}{ \#6 } & \multicolumn{2}{|c|}{$\# 7$} & \multicolumn{2}{|c|}{ \#8 } & \multicolumn{2}{|c|}{$\# 9$} & \multicolumn{2}{|c|}{$\# 10$} \\
\hline & 0.62 & 0.28 & 0.63 & 0.35 & 0.66 & 0.27 & 0.61 & 0.30 & 0.52 & 0.32 & 0.62 & 0.31 & 0.72 & 0.26 & 0.60 & 0.31 & 0.59 & 0.30 & 0.57 & 0.35 \\
\hline $25 \%-50 \%$ & 0.56 & 0.32 & 0.50 & 0.34 & 0.52 & 0.32 & 0.47 & 0.32 & 0.44 & 0.31 & 0.53 & 0.33 & 0.55 & 0.27 & 0.48 & 0.34 & 0.54 & 0.30 & 0.57 & 0.32 \\
\hline $50 \%-75 \%$ & 0.41 & 0.32 & 0.40 & 0.30 & 0.44 & 0.32 & 0.37 & 0.29 & 0.39 & 0.27 & 0.42 & 0.32 & 0.42 & 0.29 & 0.51 & 0.34 & 0.43 & 0.30 & 0.45 & 0.31 \\
\hline $75 \%-100 \%$ & 0.35 & 0.30 & 0.33 & 0.25 & 0.33 & 0.28 & 0.37 & 0.32 & 0.38 & 0.33 & 0.37 & 0.33 & 0.30 & 0.28 & 0.43 & 0.29 & 0.32 & 0.28 & 0.31 & 0.30 \\
\hline
\end{tabular}



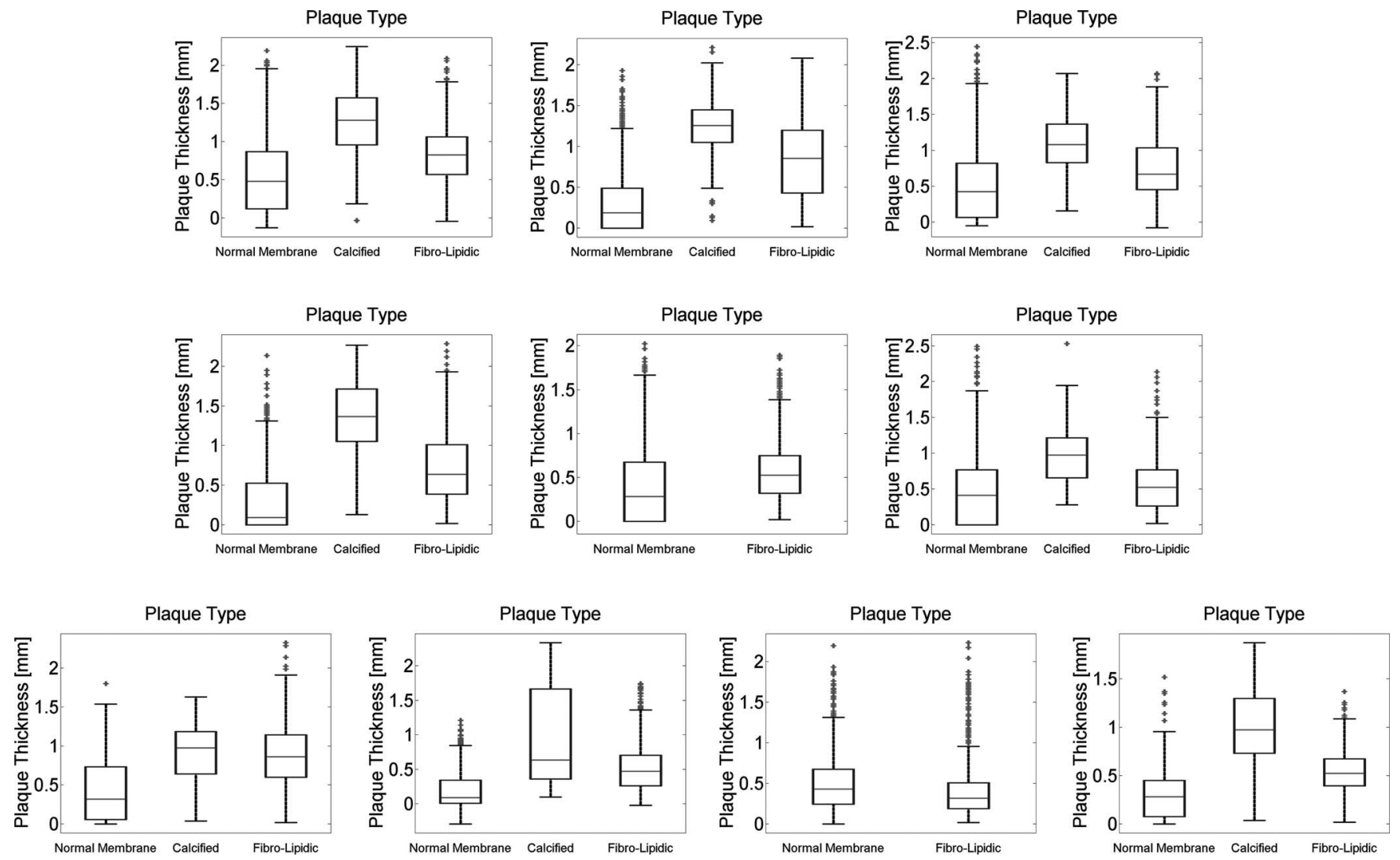

FIG. 9. Distribution of the plaque thickness according to the plaque type for the ten analyzed arteries. Cases \#5 and \#9 present no calcified plaque.

the results along the whole cross-section of the artery, the plaque presenting an higher thickness (when present) was the calcified plaque (Fig. 9 and Table II). This relation is significative in all the arteries except for the cases \#7 and \#8. As expected, the regions of fibro-lipidic plaque present a higher thickness with respect to the healthy membrane, as also confirmed by clinical observations. ${ }^{44,45}$ This relation might be explained by the fact that the calcified plaque is mostly localized in a specific eccentric angular sector of the artery, while the fibrotic region is mostly concentric and uniformly distributed.

\section{III.C.3. Wall shear stress vs plaque type}

In this experiment, the wall shear stress is globally computed (along the whole artery) for each plaque type. In Ref. 26, a similar analysis has been performed, averaging the results over 20 analyzed arteries, observing that the higher WSS values are associated with calcified regions. Unfortunately, a separated result for each artery is not reported. According to our computation, this relation is confirmed in five (cases \#2, \#4, \#7, \#8, and \#10) over eight arteries presenting calcified regions (Fig. 10 and Table III). This finding is confirmed by Wentzel ${ }^{46}$ who observed that the regions having higher WSS are located in the proximity of a localized thickest membrane, and these regions have been associated with the calcified plaque in the previous experiment (Sec. III.C.2).

\section{III.C.4. Von Mises stress vs wall shear stress}

Finally, the analysis of the relation between fluid and membrane stresses is performed at the global level. Since the biomechanical model used in the simulation obtain the plaque

TABLE II. Distribution of the plaque thickness according to the plaque type for the ten analyzed arteries (mean and std). Cases \#5 and \#9 present no calcified plaque.

\begin{tabular}{|c|c|c|c|c|c|c|c|c|c|c|c|c|c|c|c|c|c|c|c|c|}
\hline \multirow{2}{*}{$\frac{\text { Case }}{\text { Normal membrane }}$} & \multicolumn{2}{|c|}{$\# 1$} & \multicolumn{2}{|c|}{$\# 2$} & \multicolumn{2}{|c|}{$\# 3$} & \multicolumn{2}{|c|}{ \#4 } & \multicolumn{2}{|c|}{$\# 5$} & \multicolumn{2}{|c|}{ \#6 } & \multicolumn{2}{|c|}{$\# 7$} & \multicolumn{2}{|c|}{ \#8 } & \multicolumn{2}{|c|}{$\# 9$} & \multicolumn{2}{|c|}{$\# 10$} \\
\hline & 0.56 & 0.50 & 0.29 & 0.35 & 0.54 & 0.54 & 0.32 & 0.42 & 0.38 & 0.52 & 0.47 & 0.46 & 0.49 & 0.49 & 0.21 & 0.28 & 0.51 & 0.40 & 0.29 & 0.25 \\
\hline Calcified & 1.25 & 0.42 & 1.22 & 0.37 & 1.11 & 0.39 & 1.37 & 0.46 & $\ldots$ & $\ldots$ & 1.03 & 0.50 & 0.92 & 0.37 & 0.93 & 0.70 & $\cdots$ & $\ldots$ & 1.00 & 0.39 \\
\hline Fibro-lipidic & 0.83 & 0.35 & 0.85 & 0.49 & 0.76 & 0.39 & 0.72 & 0.45 & 0.59 & 0.39 & 0.59 & 0.41 & 0.87 & 0.42 & 0.54 & 0.37 & 0.40 & 0.33 & 0.53 & 0.25 \\
\hline
\end{tabular}



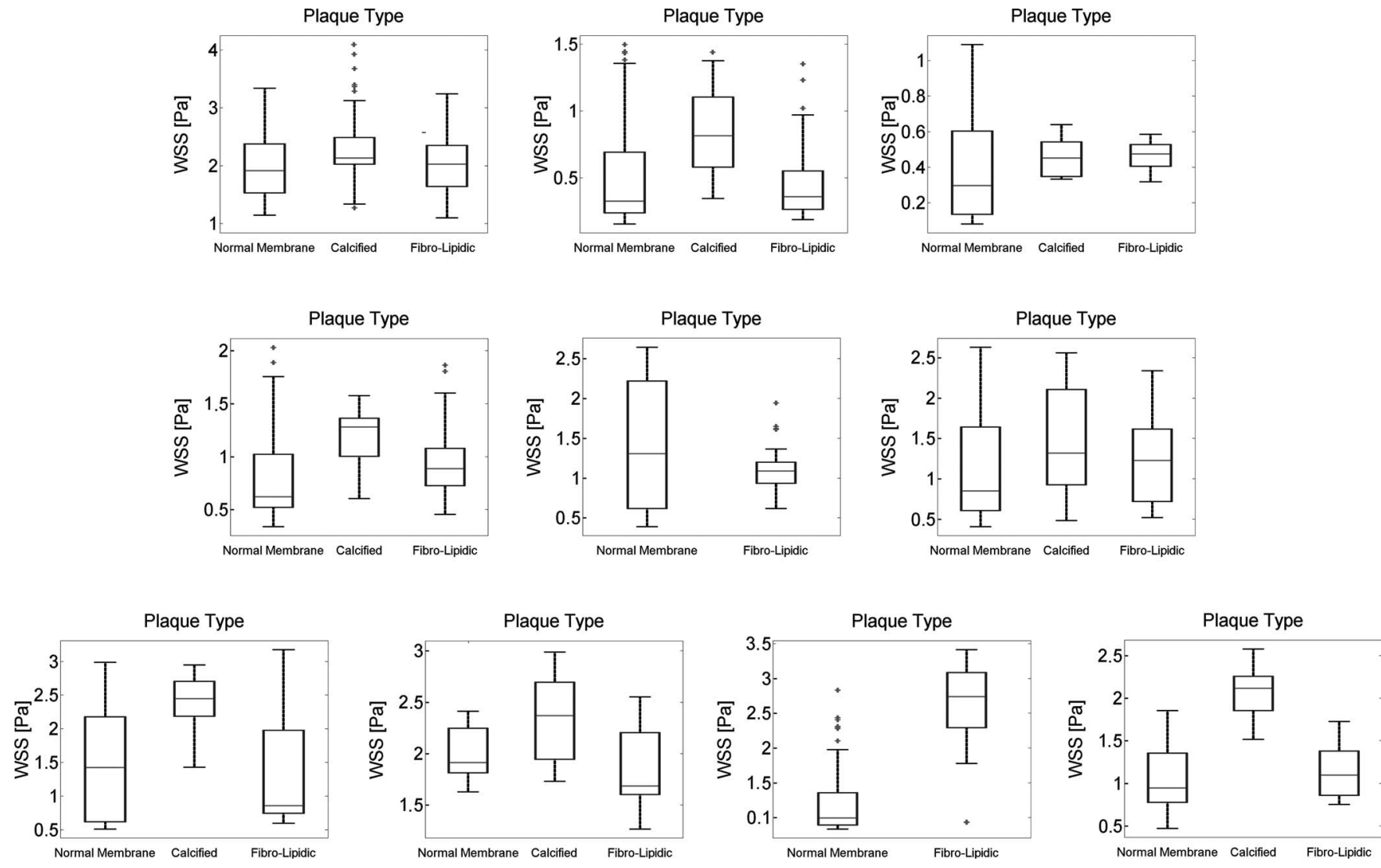

FIG. 10. Distribution of the wall shear stress according to the plaque type for the ten analyzed arteries.

stress (Von Mises Stress MAP) as function of the Plaque Type $M A P$ and Thickness MAP, these three variables are not independent, hence their relation is obvious. Instead, we report the normalized distribution of the wall shear stress as a function of the Von Mises stress intensity. The graphics in Fig. 11, supported by the statistics in Table IV, suggest a possible hyperbolic inverse relation between the two indices. However, to the best of our knowledge, there are not previous studies in vivo, providing clinical observations or direct measurements describing the relation between those quantities. The possibility to estimate the Von Mises stresses in vivo can potentially provide additional insight into the study of the plaque rupture. ${ }^{7}$ Given that no straightforward mechanical relation between the fluid stresses (WSS) and the plaque stresses (VMS) is expected, these observations suggest that further analysis should include the exploration of complementary cardiovascular risk factors (e.g., genetics, inflammatory, epidemiologic...).

\section{CONCLUSIONS AND DISCUSSION}

In this study, we introduced a framework able to provide the exhaustive assessment of morphological (vessel radius, plaque type, and thickness), fluid-dynamic (wall shear stress), and structural (Von Mises stress) indices from IVUS and angiographic sequences. In order to exploit highly reliable landmarks such as bifurcations for defining the $3 \mathrm{D}$ vessel orientation of the vessel, the tool requires minimal user interaction. However, such step could be avoided, making the approach fully automatic, by using branching identification methods ${ }^{35,36}$ or by using the optimization approach proposed in Ref. 19.

Although the vessel analysis along the full cardiac cycle would provide significant information for prediction of plaque rupture, the reconstruction of the $3 \mathrm{D}$ arterial walls along the cardiac cycle from IVUS images seems to be still an open issue. Indeed, to the best of our knowledge, the reconstruction

TABLE III. Distribution of the wall shear stress according to the plaque type for the ten analyzed arteries (mean and std).

\begin{tabular}{|c|c|c|c|c|c|c|c|c|c|c|c|c|c|c|c|c|c|c|c|c|}
\hline \multirow{2}{*}{$\begin{array}{l}\text { Case } \\
\text { Normal membrane }\end{array}$} & \multicolumn{2}{|c|}{$\# 1$} & \multicolumn{2}{|c|}{$\# 2$} & \multicolumn{2}{|c|}{ \#3 } & \multicolumn{2}{|c|}{ \#4 } & \multicolumn{2}{|c|}{ \#5 } & \multicolumn{2}{|c|}{ \#6 } & \multicolumn{2}{|c|}{ \#7 } & \multicolumn{2}{|c|}{ \#8 } & \multicolumn{2}{|c|}{ \#9 } & \multicolumn{2}{|c|}{$\# 10$} \\
\hline & 1.95 & 0.81 & 0.43 & 1.12 & 0.39 & 0.45 & 0.71 & 0.42 & 1.53 & 1.64 & 1.19 & 0.97 & 1.43 & 1.51 & 2.10 & 0.41 & 1.23 & 0.42 & 1.15 & 0.61 \\
\hline Calcified & 2.23 & 0.42 & 0.78 & 1.23 & 0.44 & 0.23 & 1.22 & 0.34 & $\ldots$ & $\ldots$ & 1.48 & 1.21 & 2.51 & 0.65 & 2.38 & 0.81 & $\ldots$ & $\ldots$ & 2.11 & 0.45 \\
\hline Fibro-lipidic & 1.99 & 0.58 & 0.41 & 0.35 & 0.48 & 0.18 & 0.85 & 0.31 & 1.18 & 0.22 & 1.25 & 0.84 & 1.48 & 1.31 & 1.83 & 0.74 & 2.71 & 0.72 & 1.23 & 0.56 \\
\hline
\end{tabular}



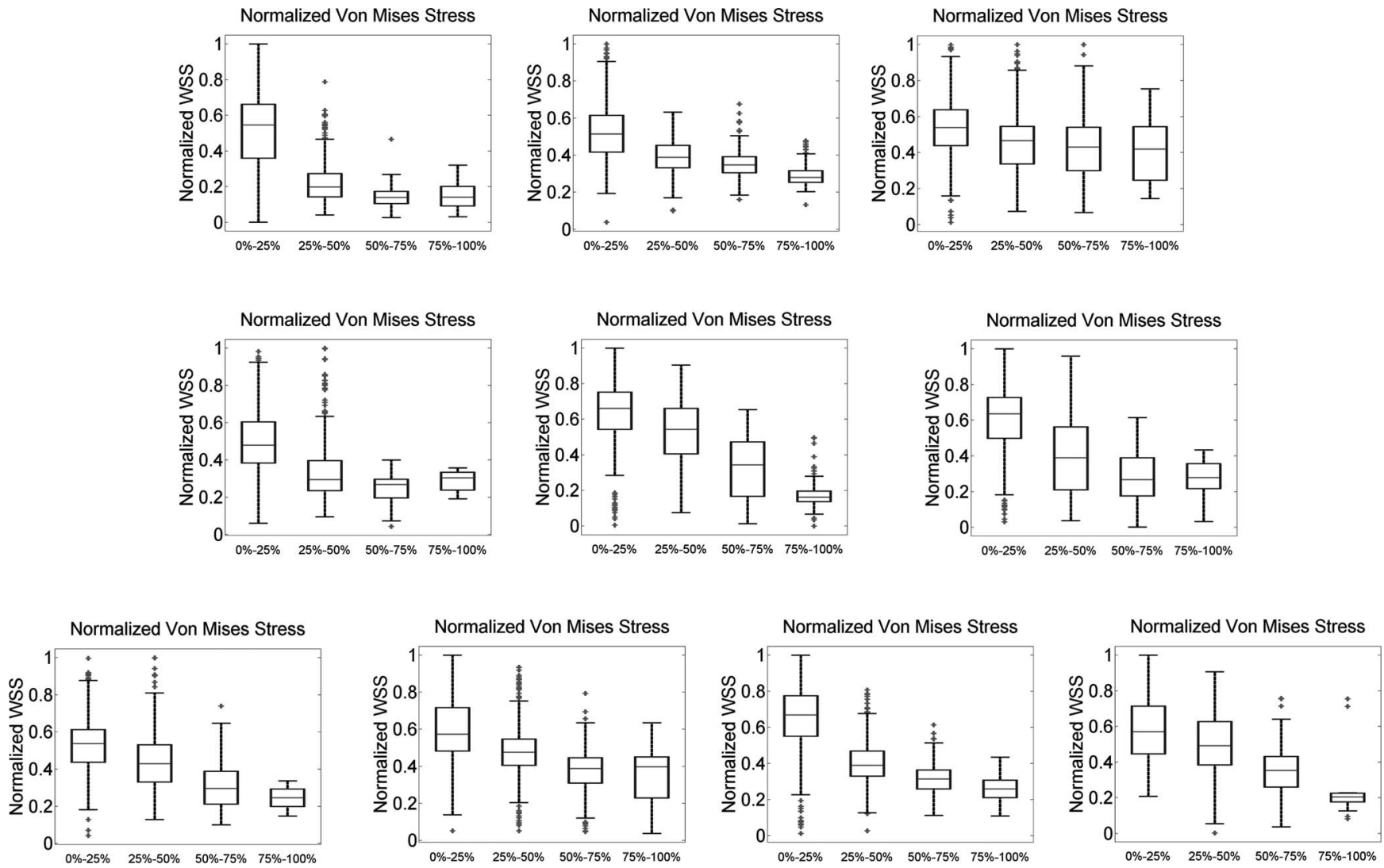

FIG. 11. Distribution of the normalized wall shear stress according to the Von Mises stress.

of the vessel along the cardiac cycle requires a specific hardware (a biplane angiography system), which has been used by Zhu and Friedman, ${ }^{1}$ Gijsen et al.,${ }^{20} \mathrm{Krams}$ et al. ${ }^{18}$ Wahle et al. ${ }^{19}$ and Samady et al. ${ }^{26}$ However, all the authors (with the exception of Zhu and Friedman ${ }^{1}$ ) studied only a single 3D geometry (gated or averaged) and not the full dynamic of the cardiac cycle. Since the biplane angiography system is poorly available in hospitals, the reconstruction framework presented in this paper employs a standard one plane x-ray system, making the approach easily reproducible in any hospital facility.

Since the purpose of the paper is to give a general estimation of the relation between arterial properties, rather than a detailed simulation of the vessel behavior, some classification and modeling simplifications have been considered. Indeed, as future work, the IVUS tissue properties will be extended, discriminating the plaque area into a wider number of categories (normal membrane, fibrotic, lipidic, calcified, necrotic core); the depth and the relative amount of each plaque, will be further analyzed, allowing a much richer anal- ysis of the coronary artery and the plaque rupture risk. Additionally, such volumetric measurements will allow a volumetric definition of the plaque (identifying, for instance, lipidic pools, and fibrotic thin caps) required for more complex modeling of the arterial wall. ${ }^{2}$ The vessel membrane, which has been defined in this paper as a shell object (with isotropic, elastic properties, and composed by Argyris elements), could be redesigned using anisotropic, hyperelastic, or visco-elastic models and computed using a full-volumetric meshing. However, we would like to stress that the simplified membrane design allowed to speed up about 60 times the computation of the structural stress. According to our experiments, when a single tissue type is used, the error committed computing the Von Mises stress using a simplified shell model instead of a full 3D mesh is about 1\%, and hence negligible with respect to the conclusion shown in this paper. The fluid modeling could benefit by the use of advanced rheological laws, such as the Power Law, describing the vessel profile as a function of the blood viscosity. As a matter of fact, fluid-dynamic indices such as velocity profile and streamline could be

TABLE IV. Distribution of the normalized wall shear stress according to the Von Mises stress (mean and std).

\begin{tabular}{|c|c|c|c|c|c|c|c|c|c|c|c|c|c|c|c|c|c|c|c|c|}
\hline \multirow{2}{*}{$\frac{\text { Case }}{0 \%-25 \%}$} & \multicolumn{2}{|c|}{$\# 1$} & \multicolumn{2}{|c|}{$\# 2$} & \multicolumn{2}{|c|}{ \#3 } & \multicolumn{2}{|c|}{$\# 4$} & \multicolumn{2}{|c|}{ \#5 } & \multicolumn{2}{|c|}{ \#6 } & \multicolumn{2}{|c|}{$\# 7$} & \multicolumn{2}{|c|}{$\# 8$} & \multicolumn{2}{|c|}{$\# 9$} & \multicolumn{2}{|c|}{$\# 10$} \\
\hline & 0.22 & 0.04 & 0.22 & 0.04 & 0.17 & 0.02 & 0.23 & 0.03 & 0.16 & 0.02 & 0.24 & 0.03 & 0.24 & 0.04 & 0.18 & 0.02 & 0.20 & 0.02 & 0.18 & 0.02 \\
\hline $25 \%-50 \%$ & 0.17 & 0.02 & 0.19 & 0.02 & 0.15 & 0.02 & 0.20 & 0.03 & 0.15 & 0.02 & 0.21 & 0.03 & 0.22 & 0.04 & 0.17 & 0.01 & 0.17 & 0.01 & 0.17 & 0.02 \\
\hline $50 \%-75 \%$ & 0.16 & 0.01 & 0.18 & 0.02 & 0.15 & 0.02 & 0.18 & 0.01 & 0.13 & 0.01 & 0.19 & 0.02 & 0.19 & 0.03 & 0.16 & 0.01 & 0.16 & 0.01 & 0.15 & 0.01 \\
\hline $75 \%-100 \%$ & 0.16 & 0.01 & 0.16 & 0.02 & 0.15 & 0.02 & 0.19 & 0.01 & 0.11 & 0.01 & 0.19 & 0.02 & 0.17 & 0.01 & 0.16 & 0.01 & 0.15 & 0.01 & 0.15 & 0.02 \\
\hline
\end{tabular}


integrated in the approach, for instance, coupled to LDL deposit analyses. ${ }^{2}$ However, it is interesting to note that each module of the framework could be easily replaced by a more sophisticated analysis.

Presently, the reconstruction process has to be performed offline (and not interoperatively), because of the computational complexity of the algorithms. The lumen and media segmentations require about 5 and 2 seconds per frame respectively (i.e., 12 minutes per pullback), using a MATLAB nonoptimized code. The frame rotation alignment stage takes 0.3 seconds per frame. The fluid dynamic computation requires approximately $3 \mathrm{~min}$, while the structural analysis (after the speed up due to the model simplification) about 4 min on a dual-core Intel Pc. The vessel reconstruction is almost real-time.

In this paper, the relation between the computed arterial indices is qualitatively and quantitatively analyzed, in ten coronary arteries, in order to illustrate the feasibility of the pipeline. The measurements confirmed observations of previous clinical and technical studies, , 18,19,22,26,46 thus illustrating the capabilities of the tool.

Although in the present paper, a fewer number of plaque types with respect to Ref. 26 are considered, each experiment additionally reports the statistics for the healthy membrane. Moreover, as shown in Sec. III.C.2, the analysis of the vessel is more detailed since it is performed considering independently the sectorial samples, instead of averaging the results per cross-sections. Finally, for the first time, the inverse relation between fluid-dynamics and wall stresses has been studied on coronary in vivo data. The study of additional arterial indices, such as flexional stress, will be considered as future research.

It is interesting to note that in the experiments presented in Secs. III.C. 2 and III.C.3, the relationships observed for some artery slightly deviate from the general behavior of the patient population, showing the interest of a separated analysis for each patient. The interpretation of the results for such outliers is currently an open issue, since it requires the extension of the study to a larger clinical data-set. Indeed, the aim of this paper is to show a feasibility study, while further investigations on a statistically relevant patient data-base will allow to draw solid and novel clinical findings.

The current study is limited by mixing early-stage atheromas, which do not alter the lumen geometry due to compensatory (positive) remodeling, with advanced atheromas where the lumen geometry has been altered by luminal narrowing already, thus distorting the shear-stress assessment to that extent. In particular, the comparison between different stages of atherosclerosis (positive and negative remodeling), including follow-up cases, will be crucial to investigate the evolution of the arterial disease and the assessment of risk of rupture in vulnerable plaque.

\section{ACKNOWLEDGMENTS}

The authors would like to thank Dr. Fina Mauri of the hospital "Germans Trias i Pujol," Badalona, Spain for providing the clinical cases used in this study and for her support in the project. They would also like to acknowledge Dr. Francesco Ciompi and Dr. Oriol Pujol for their suggestions and for providing the code of the media-aventitia segmentation method.

This work has been supported in part by the projects TIN2009-14404-C02, La Marató de TV3 082131, CONSOLIDER INGENIO CSD 2007-00018, AIB2010SE-00210 of the Spanish Ministry of Science and Innovation (MICINN), "Lumen Border Detection" project in collaboration with Boston Scientific Scimed, Inc., USA and SGR00696. The work of C. Gatta is supported by the Spanish Ministry of Science and Innovation MICINN under a Ramon y Cajal Research Fellowship.

\footnotetext{
a)Electronic mail: balocco.simone@gmail.com
}

${ }^{1}$ H. Zhu and M. H. Friedman, "Relationship between the dynamic geometry and wall thickness of a human coronary artery," Arterioscler., Thromb., Vasc. Biol. 23(12), 2260-2265 (2003).

${ }^{2}$ P. Siogkas, A. Sakellarios, T. P. Exarchos, L. Athanasiou, E. Karvounis, K. Stefanou, E. Fotiou, D. I. Fotiadis, K. K. Naka, L. K. Michalis, N. Filipovic, and O. Parodi, "Multiscale-patient-specific artery and atherogenesis models," IEEE Trans. Biomed. Eng. 58(12), 3464-3468 (2011).

${ }^{3}$ R. Baldewsing, C. DeKorte, J. Schaar, F. Mastik, and A. V. d. Steen, "Finite element modeling and intravascular ultrasound elastography of vulnerable plaques: Parameter variation," Ultrasonics 42, 723-729 (2004).

${ }^{4}$ A. I. Veress, D. G. Vince, P. M. Anderson, J. F. Cornhill, E. E. Herderick, J. D. Klingensmith, B. D. Kuban, N. L. Greenberg, and J. D. Thomas, "Vascular mechanics of the coronary artery," Z. Kardiol. 89(2), 92-100 (2000).

${ }^{5}$ S. D. Ramaswamy, S. C. Vigmostad, A. Wahle, Y. G. Lai, M. E. Olszewski, K. C. Braddy, T. M. Brennan, J. D. Rossen, M. Sonka, and K. B. Chandran, "Fluid dynamic analysis in a human left anterior descending coronary artery with arterial motion," Ann. Biomed. Eng. 32(12), 16281641 (2004).

${ }^{6} \mathrm{~J}$. D. Humphrey, "Mechanisms of arterial remodeling in hypertension. coupled roles of wall shear and intramural stress," Hypertension 52, 195-200 (2008).

${ }^{7}$ D. Tang, Z. Teng, G. Canton, C. Yang, M. Ferguson, X. Huang, J. Zheng, P. K. Woodard, and C. Yuan, "Sites of rupture in human atherosclerotic carotid plaques are associated with high structural stresses: An in vivo mri-based 3d fluid-structure interaction study," Stroke 40(10), 3258-3263 (2009).

${ }^{8}$ S. Balocco, C. Gatta, X. Carrillo, J. Mauri, and P. Radeva, "Plaque type, plaque burden, and wall shear stress relation in coronary arteries assessed by x-ray angiography and intravascular ultrasound: a qualitative study," in ISABEL'11 Proceedings of the 4th International Symposium on Applied Sciences in Biomedical and Communication Technologies, Barcelona, Spain (ACM, New York, 2011).

${ }^{9}$ F. Ciompi, O. Pujol, C. Gatta, X. Carrillo, J. M. Ferre, and P. Radeva, "A holistic approach for the detection of media-adventitia border in ivus," in Proceedings of the 14th International Conference on Medical Image Computing and Computer-assisted Intervention, Barcelona, Spain (Springer-Verlag, Berlin, Heidelberg, 2011), Vol. Part III, pp. 411-419.

${ }^{10}$ F. Ciompi, O. Pujol, C. Gatta, O. Rodriguez-Leor, J. Mauri, and P. Radeva, "Fusing in-vitro and in-vivo intravascular ultrasound data for plaque characterization,” Int. J. Cardiovasc. Imaging 26(7), 763-779 (2010).

${ }^{11}$ H. Huang, R. Virmani, H. Younis, A. P. Burke, R. D. Kamm, and R. T. Lee, "The impact of calcification on the biomechanical stability of atherosclerotic plaques," Circulation 103(8), 1051-1056 (2001).

${ }^{12}$ C. J. Slager, J. J. Wentzel, J. C. Schuurbiers, J. A. Oomen, J. Kloet, R. Krams, C. von Birgelen, W. J. van der Giessen, P. W. Serruys, and P. J. de Feyter, "True 3-dimensional reconstruction of coronary arteries in patients by fusion of angiography and ivus (angus) and its quantitative validation," Circulation 102(5), 511-516 (2000).

${ }^{13}$ A. Wahle, M. E. Olszewski, and M. Sonka, "Interactive virtual endoscopy in coronary arteries based on multimodality fusion," IEEE Trans. Med. Imaging 23(11), 1391-403 (2004).

${ }^{14}$ K. R. Hoffmann, A. Wahle, C. Pellot-Barakat, J. Sklansky, and M. Sonka, "Biplane x-ray angiograms, intravascular ultrasound, and 3d visualization of coronary vessels," Int. J. Cardiovasc. Imaging 15(6), 495-512 (1999). 
${ }^{15}$ J. Dijkstra and S. Carlier, "Accurate plaque volume measurements in $3 \mathrm{~d}$ reconstructed ivus pullback sequences," Int. J. Cardiovasc. Imaging 19(4), 313-314 (2003).

${ }^{16}$ C. V. Bourantas, I. C. Kourtis, M. E. Plissiti, D. I. Fotiadis, C. S. Katsouras, M. I. Papafaklis, and L. K. Michalis, "A method for 3d reconstruction of coronary arteries using biplane angiography and intravascular ultrasound images," Comput. Med. Imaging Graph. 29(8), 597-606 (2005).

${ }^{17}$ D. Rotger, M. Rosales, J. Garcia, O. Pujol, J. Mauri, and P. Radeva, "Active vessel: A new multimedia workstation for intravascular ultrasound and angiography fusion," Comput. Cardiol. 30, 65-68 (2003).

${ }^{18}$ R. Krams, J. J. Wentzel, J. A. Oomen, R. Vinke, J. C. Schuurbiers, P. J. de Feyter, P. W. Serruys, and C. J. Slager, "Evaluation of endothelial shear stress and $3 \mathrm{~d}$ geometry as factors determining the development of atherosclerosis and remodeling in human coronary arteries in vivo. combining $3 \mathrm{~d}$ reconstruction from angiography and ivus (angus) with computational fluid dynamics," Arterioscler., Thromb., Vasc. Biol. 17(10), 2061-2065 (1997).

${ }^{19}$ A. Wahle, J. J. Lopez, M. E. Olszewski, S. C. Vigmostad, K. B. Chandran, J. D. Rossen, and M. Sonka, "Plaque development, vessel curvature, and wall shear stress in coronary arteries assessed by $\mathrm{x}$-ray angiography and intravascular ultrasound," Med. Image Anal. 10(4), 615-631 (2006).

${ }^{20}$ F. J. Gijsen, J. J. Wentzel, A. Thury, F. Mastik, J. A. Schaar, J. C. Schuurbiers, C. J. Slager, W. J. van der Giessen, P. J. de Feyter, A. F. van der Steen, and P. W. Serruys, "Strain distribution over plaques in human coronary arteries relates to shear stress," Am. J. Physiol. Heart Circ. Physiol. 295(4), H1608-H1614 (2008).

${ }^{21}$ A. G. van der Giessen, M. Schaap, F. J. Gijsen, H. C. Groen, T. van Walsum, N. R. Mollet, J. Dijkstra, F. N. van de Vosse, W. J. Niessen, P. J. de Feyter, A. F. van der Steen, and J. J. Wentzel, "3d fusion of intravascular ultrasound and coronary computed tomography for in-vivo wall shear stress analysis: a feasibility study," Int. J. Cardiovasc. Imaging 26(7), 781-796 (2009).

${ }^{22}$ J. F. LaDisa, M. Bowers, L. Harmann, R. Prost, A. V. Doppalapudi, T. Mohyuddin, O. Zaidat, and R. Q. Migrino, "Time-efficient patient-specific quantification of regional carotid artery fluid dynamics and spatial correlation with plaque burden," Med. Phys. 37(2), 784-792 (2010).

${ }^{23} \mathrm{~S}$. Chen and J. Carroll, "Kinematic and deformation analysis of 4-d coronary arterial trees reconstructed from cine angiograms," IEEE Trans. Med. Imaging 22(6), 710-721 (2003).

${ }^{24}$ E. Hansis, J. Carroll, D. Schafer, O. Dossel, and M. Grass, "High-quality 3d coronary artery imaging on an interventional c-arm x-ray system," Med. Phys. 37(4), 1601-1610 (2010).

${ }^{25} \mathrm{H}$. Wu, S. Chen, S. Shroff, and J. Carroll, "Stress analysis using anatomically realistic coronary tree," Med. Phys. 30(11), 2927-2936 (2003).

${ }^{26}$ H. Samady, P. Eshtehardi, M. C. McDaniel, J. Suo, S. S. Dhawan, C. Maynard, L. H. Timmins, A. A. Quyyumi, and D. P. Giddens, "Coronary artery wall shear stress is associated with progression and transformation of atherosclerotic plaque and arterial remodeling in patients with coronary artery disease," Circulation 124(7), 779-788 (2011).

${ }^{27}$ T. Thim, M. K. Hagensen, D. Wallace-Bradley, J. F. Granada, G. L. Kaluza, L. Drouet, W. P. Paaske, H. E. Botker, and E. Falk, "Unreliable assessment of necrotic core by virtual histology intravascular ultrasound in porcine coronary artery disease," Circ. Cardiovasc. Imaging 3(4), 384-391 (2010).

${ }^{28}$ S. Balocco, C. Gatta, F. Ciompi, O. Pujol, X. Carrillo, J. Mauri, and P. Radeva, "Combining growcut and temporal correlation for ivus lumen segmentation," in IbPRIA'11 Proceedings of the 5th Iberian Conference on Pattern Recognition and Image Analysis, Gran Canaria, Spain (SpringerVerlag, Berlin, 2011), pp. 100-108.

${ }^{29}$ C. Gatta, S. Balocco, F. Ciompi, R. Hemetsberger, O. Rodriguez Leor, and P. Radeva, "Real-time gating of ivus sequences based on motion blur anal- ysis: Method and quantitative validation," Med. Image Comput. Comput. Assist. Interv. 13(2), 59-67 (2010).

${ }^{30}$ V. Vezhnevets and V. Konouchine, "Grow-cut" - interactive multi-label n-d image segmentation," Proc. Graphicon 2005, 150-156.

${ }^{31}$ F. Ciompi, O. Pujol, C. Gatta, M. Alberti, S. Balocco, X. Carrillo, J. MauriFerre, and P. Radeva, "Holimab: A holistic approach for media-adventitia border detection in intravascular ultrasound," Med. Image Anal. 16, 10851100 (2012).

${ }^{32}$ C. Gatta, E. Puertas, and O. Pujol, "Multi-scale stacked sequential learning," Pattern Recogn. 44, 2414-2426 (2011).

${ }^{33}$ L. Cohen and R. Kimmel, "Global minimum for active contour models: A minimal path approach,” Int. J. Comput. Vis. 24, 57-78 (1997).

${ }^{34}$ C. Canero, F. Vilarino, J. Mauri, and P. Radeva, "Predictive (un)distortion model and 3-d reconstruction by biplane snakes," IEEE Trans. Med. Imaging 21(9), 1188-1201 (2002).

${ }^{35}$ M. Alberti, S. Balocco, C. Gatta, F. Ciompi, O. Pujol, J. Silva, X. Carrillo, and P. Radeva, "Automatic bifurcation detection in coronary ivus sequences," IEEE Trans. Biomed. Eng. 59(4), 1022-1031 (2012).

${ }^{36}$ G. Azzopardi and N. Petkov, "Detection of retinal vascular bifurcations by trainable v4-like filters," in Proceedings of the 14th International Conference on Computer Analysis of Images and Patterns, Seville, Spain (Springer, Berlin, 2011), Vol. I, pp. 451-459.

${ }^{37}$ C. Gatta, O. Pujol, O. Rodriguez-Leor, J. Mauri, and P. Radeva, "Fast rigid registration of vascular structures in ivus sequences," IEEE Trans. Inf. Technol. Biomed. 13, 1006-1011 (2009).

${ }^{38}$ S. D. Ramaswamy, S. C. Vigmostad, A. Wahle, Y. G. Lai, M. E. Olszewski, K. C. Braddy, T. M. Brennan, J. D. Rossen, M. Sonka, and K. B. Chandran, "Comparison of left anterior descending coronary artery hemodynamics before and after angioplasty," J. Biomech. Eng. 128(1), 40-48 (2006).

${ }^{39}$ R. W. Downe, A. Wahle, J. W. Garvin, T. Kovarnik, J. Horak, J. J. Lopez, and M. Sonka, "Identification and 3-d modeling of coronary branches in intravascular ultrasound," MICCAI Workshop in Computing and Visualization for (Intra)Vascular Imaging (CVII) (Springer, Toronto, 2011).

${ }^{40} \mathrm{~S}$. Balocco, O. Camara, E. Vivas, T. Sola, L. Guimaraens, H. A. Gratama van Andel, C. B. Majoie, J. M. Pozo, B. H. Bijnens, and A. F. Frangi, "Feasibility of estimating regional mechanical properties of cerebral aneurysms in vivo," Med. Phys. 37(4), 1689-1706 (2010).

${ }^{41}$ J. H. Argyris, I. Fried, and D. W. Scharpf, "The tuba family of plate elements for the matrix displacement method," J. R. Aeronaut. Soc. 72, 701-709 (1968).

${ }^{42}$ R. A. Baldewsing, C. L. de Korte, J. A. Schaar, F. Mastik, and A. F. van der Steen, "A finite element model for performing intravascular ultrasound elastography of human atherosclerotic coronary arteries," Ultrasound Med. Biol. 30(6), 803-813 (2004).

${ }^{43}$ H. M. Garcia-Garcia, G. S. Mintz, A. Lerman, D. G. Vince, M. P. Margolis, G. A. van Es, M. A. Morel, A. Nair, R. Virmani, A. P. Burke, G. W. Stone, and P. W. Serruys, "Tissue characterisation using intravascular radiofrequency data analysis: Recommendations for acquisition, analysis, interpretation and reporting," EuroIntervention 5(2), 177-189 (2009).

${ }^{44}$ T. Kataoka, V. Mathew, R. Rubinshtein, C. S. Rihal, R. Lennon, L. O. Lerman, and A. Lerman, "Association of plaque composition and vessel remodeling in atherosclerotic renal artery stenosis: A comparison with coronary artery disease," JACC Cardiovasc. Imaging 2(3), 327-338 (2009).

${ }^{45}$ J. Schoepf, "Coronary calcium scanning," CT of the Heart: Principles and Applications, 1st ed. (Humana, 2004).

${ }^{46}$ J. J. Wentzel, E. Janssen, J. Vos, J. C. Schuurbiers, R. Krams, P. W. Serruys, P. J. de Feyter, and C. J. Slager, "Extension of increased atherosclerotic wall thickness into high shear stress regions is associated with loss of compensatory remodeling," Circulation 108(1), 17-23 (2003). 